LBNL-39652

\title{
Detectability of Strange Matter in Heavy Ion Experiments
}

\author{
Jürgen Schaffner-Bielich ${ }^{a, b, c}$, Carsten Greiner ${ }^{d}$, Alexander Diener ${ }^{b}$ and Horst Stöcker ${ }^{b}$ \\ a The Niels Bohr Institute, Blegdamsvej 17, DK-2100 Copenhagen, Denmark \\ ${ }^{b}$ Institut für Theoretische Physik, J.W. Goethe-Universität, D-60054 Frankfurt, Germany \\ ${ }^{c}$ Nuclear Science Division, LBNL, Berkeley, 94720 California \\ ${ }^{d}$ Institut für Theoretische Physik, Justus-Liebig Universität, D-35392 Giessen, Germany
}

(November 22, 1996)

\begin{abstract}
We discuss the properties of two distinct forms of hypothetical strange matter, small lumps of strange quark matter (strangelets) and of hyperon matter (metastable exotic multihypernuclear objects: MEMOs), with special emphasis on their relevance for present and future heavy ion experiments. The masses of small strangelets up to $A_{B}=40$ are calculated using the MIT bag model with shell mode filling for various bag parameters. The strangelets are checked for possible strong and weak hadronic decays, also taking into account multiple hadron decays. It is found that strangelets which are stable against strong decay are most likely highly negative charged, contrary to previous findings. Strangelets can be stable against weak hadronic decay but their masses and charges are still rather high. This has serious impact on the present high sensitivity searches in heavy ion experiments at the AGS and CERN facilities. On the other hand, highly charged MEMOs are predicted on the basis of an extended relativistic mean-field model. Those objects could be detected in future experiments searching for short-lived, rare composites.
\end{abstract}


It is demonstrated that future experiments can be sensitive to a much wider variety of strangelets.

\section{INTRODUCTION}

One of the most fascinating aspects of modern particle physics is the phase transition from hadronic matter to a deconfined strong interacting plasma phase. New forms of matter might be possible [1] and formed during this transition. It has been proposed that the introduction of strange quarks into a plasma with two flavours could lower the Fermi energy of the system and thus the mass of the quark matter. If its mass is lower than the mass of hadronic (hyperonic) matter with the same strangeness fraction, it could not decay (completely) via strong interactions, which means that it would be metastable [2]. If its mass is even lower than nonstrange nucleonic matter, quark matter would be the true ground state of nuclear matter [3].

The only way to produce a quark-gluon plasma in the laboratory are collisions of heavy ions at ultrarelativistic energies. It has been shown that during the stage of coexistence of the two phases the abundantly produced strange and antistrange quarks are distributed asymmetrically between the quark and the hadron phase [4,5]. The strange quarks are enriched in the plasma, thus lowering its mass, whereas the antistrange quarks are found predominantly in the hadronic sector. Radiated pions and kaons carry away entropy and antistrangeness from the system, thus cooling it and charging the quark droplet with net strangeness. This mechanism can lead to the formation of a droplet of rather cold, strange quark matter, a strangelet.

On the other hand, even without a phase transition strangeness and antistrangeness is abundantly produced in heavy ion collisions. Therefore baryonic objects with a high strangeness fraction may be formed, so-called metastable exotic multihypernuclear objects or MEMOs [6]. They are expected to be bound by energies up to $E_{B} / A \approx-22 \mathrm{MeV}$ and to possess properties quite similar to those of strangelets [7]. 
There are several experiments under way at the AGS in Brookhaven using forward spectroscopy [8 11] or an open geometry [12] looking for long-lived charged strangelets $(\tau>10-100 \mathrm{~ns})$ as a 'smoking gun' for the formation of a QGP. The NEWMASS collaboration (experiment NA52) has recently reported new limits for producing long-lived strangelets $\left(\tau>10^{-6} \mathrm{~s}\right)$ at the higher bombarding energy of the SPS facility at CERN [13]. The H-dibaryon [14] with quark content (uuddss), is possibly the lightest strangelet. The search for the $\mathrm{H}$-dibaryon in the collisions of heavy ions opened a very active field of research [15]. Most recently experiment E888 at AGS set new limits on the production of the $\mathrm{H}$-dibaryon for lifetimes of $\tau>1 \mathrm{~ns}[16]$. Also quite recently experiment E896 has been approved looking for short-lived $\mathrm{H}$-dibaryons in forward direction [17] sensitive to lifetimes of $\tau \approx 10^{-11} \mathrm{~s}$.

Simple coalescence estimates give production probabilities of strange clusters of the order of $10^{3-A_{B}-|S|}$, where $S$ denotes the strangeness and $A_{B}$ the baryon number of the cluster [2.18]. Hence, small clusters with $A_{B}+|S| \leq r+3$, where $r$ is the sensitivity of the apparatus (presently $r \leq 12$ ), are most favoured for detection. Therefore, if strangelets are formed due to this scenario, baryon numbers of $A_{B} \leq 12$ are expected. Dynamical calculations with non-equilibrium particle emission suggest also $A_{B} \approx 10-30$ [5, [19] for initial entropies corresponding to AGS and SPS bombarding energies. At higher energy, at RHIC and LHC colliders, strangelet distillation still works but lower mass numbers of $A<10$ are expected [20], which might be detectable with the ALICE detector at the LHC [21].

In the following we examine the properties of both forms of strange matter for this mass range, its decay properties and its detectability for recent and future heavy ion experiments.

\section{STRANGELETS}

In the present investigation, strangelets are treated as noninteracting fermions within the MIT-Bag model, filling up the bag with exact single-particle Dirac states following [22, (4. We point out that the simple mass formula of Berger and Jaffe [23] or Fermi gas models 
including a curvature term [24] are not appropriate for describing the low mass strangelets of interest here as shell effects get crucial for their stability. Similar shell model calculations have been performed recently [25]. The authors consider strong and weak decay by nucleon and hyperon emission together and conclude that stable low mass strangelets $\left(A_{B}<100\right)$ exist which have a low (and positive) charge to mass ratio. A detailed inspection of Fig. 2 in [25] shows that strangelets with mass number $A_{B}=10$ and $Z=-4$ or $Z=7$ exist which have a rather high mass to charge ratio contrary to their conclusion. We point out that the procedure of their work is to start in the absolute minimum for a given baryon number and then to look for its stability against possible weak and strong decays. Although this investigation is very important, we believe that their finding is not fully consistent with the initial condition of possible strangelet production in relativistic heavy ion collisions. Initially, all kinds of strangelets might be produced with different quark contents. They are decaying first by strong decays (strong hadron emission) and afterwards, if surviving, by weak hadronic decays. In Ref. [25] the authors start only with those strangelets at its minimum value in $E / A$ for a given baryon number $A$ and study the stability of those candidates only. However, it is only the slowest decay, the weak leptonic decay, which ultimately drives a strangelet to its minimum value. For the purpose of detecting strangelets the direct strong decays and weak hadronic decays are now relevant, thus giving raise to a much wider class of possible candidates to be observed (see also the discussion below). In the following we recalculate strangelets for low masses and check now separately for strong and weak hadronic decay starting with all possible combinations and allowing for all kind of decays.

The MIT bag model used includes only the quark kinetic energy, the Pauli principle and confinement. It should be not taken too seriously as it effectively models the features of QCD at the confinement energy scale. It can not fix the overall energy scale due to the unambiguity of the bag constant $B$, which decides whether or not strange quark matter is stable. It does, however, illustrate potentially interesting effects as shell closure effects. Infinite strange quark matter, treated as a gas of noninteracting quarks, is absolutely stable 
for bag constants of $B^{1 / 4} \approx 145 \mathrm{MeV}$. For bag constants between $B^{1 / 4} \approx 150-200 \mathrm{MeV}$ strange quark matter is metastable, i.e. it can decay weakly depending on its strangeness content. For larger bag constants strange quark matter is unstable and decays completely via strong reactions into hadrons. Hence, we will discuss our results for various choices of the bag constant, preferably for the case of metastability.

The Dirac equation with a Bogolyubov-type boundary condition reads

$$
j_{l_{\kappa}}(p R)=-\operatorname{sgn}(\kappa) \frac{p}{E+m_{i}} j_{l_{-\kappa}}(p R)
$$

with

$$
p=\frac{\omega_{\kappa, \alpha}^{i}}{R}
$$

and the energy

$$
E_{\kappa, \alpha}^{i}=\left(\left(\frac{\omega_{\kappa, \alpha}^{i}}{R}\right)^{2}+m_{i}^{2}\right)^{1 / 2}
$$

Here $m_{i}$ denotes the mass of the quark of flavour $i$. We choose $m_{u}=m_{d}=0 \mathrm{MeV}$ and $m_{s}=150 \mathrm{MeV} . R$ is the radius of the bag, $\kappa$ the angular momentum quantum number and $\alpha$ labels the eigenvalues in the quantum state $\kappa$. The total energy is calculated by summing the lowest occupied single particle energies and adding the phenomenological bag energy $B V$ with the volume of the bag $V$, which has to be chosen such that the inside pressure of the quarks is in equilibrium with the outside vacuum pressure. The term

$$
E_{\mathrm{Cb}}=\frac{1}{15} \alpha \frac{\left(2 A_{u}-A_{d}-A_{s}\right)^{2}}{R}
$$

with the quark number $A_{i}$ of flavour $i$ accounts for Coulomb corrections. Pressure equilibrium is achieved by minimizing the total energy with respect to the bag radius. This exact result has recently been approximated by curvature contributions to the mass of strangelets [24].

We calculate the binding energy of strangelets for an arbitrary number of $u^{-}, d_{-}^{-}$, and $s$-quarks with $A_{B} \leq 16$ for a given bag parameter. Afterwards we look for possible strong 
decays, i.e. single baryon $\left\{\mathrm{n}, \mathrm{p}, \Lambda, \Sigma^{+,-}, \Xi^{0,-}, \Omega^{-}\right\}$emission and mesonic $\left\{\pi^{+,-}, K^{+,-}, K^{0}, \bar{K}^{0}\right\}$ decays (note that kaon decays do not occur in our calculation) extending the ideas already presented in [2] to finite size configuration. Note that for smaller strangelets shell effects are therefore of crucial importance. For example a strong neutron decay of a strangelet

$$
Q\left(A_{B}, S, Z\right) \rightarrow Q\left(A_{B}-1, S, Z\right)+n
$$

is allowed if the energy balance of the corresponding reaction is

$$
E\left(A_{B}, S, Z\right)>E^{\prime}\left(A_{B}-1, S, Z\right)+m_{n}
$$

where $E$ stands for the total energy of a strangelet. Multiple hadron emission is implemented by considering the combination of hadrons for a given charge $f_{z}=Z / A_{B}$ and strangeness fraction $f_{s}=|S| / A_{B}$ with the lowest total mass. The possible area for strangelets $\left(f_{s} \geq 0\right.$, $f_{z} \geq-1$, and $f_{s}+f_{z} \leq 2$ ) is divided into 9 areas of (free) hadronic matter where 7 areas are covered by the possible metastable combinations of three different baryon species as given in Table 4 of Ref. [6]. The remaining two are $\left\{\pi^{+} p \Sigma^{+}\right\}$and $\left\{\pi^{-} n \Sigma^{-}\right\}$matter. Multiple hadron emission is allowed if

$$
E\left(f_{s}, f_{z}\right)>H\left(f_{s}, f_{z}\right)
$$

where $H\left(f_{s}, f_{z}\right)$ stands for the lowest mass of hadrons for a given strangeness and charge fraction. Note that the baryon number does not enter here as it is a conserved quantity. In addition, we checked also for fission of a strangelet into another strangelet and an arbitrary number of hadrons while conserving charge, strangeness and baryon number

$$
E\left(A_{B}, S, Z\right)>E^{\prime}\left(A_{B}^{\prime}, S^{\prime}, Z^{\prime}\right)+\left(A_{B}-A_{B}^{\prime}\right) \cdot H\left(f_{s}^{\prime}, f_{z}^{\prime}\right)
$$

by three combined loops where $f_{s}^{\prime}=\left(S-S^{\prime}\right) /\left(A_{B}-A_{B}^{\prime}\right)$ and $f_{z}^{\prime}=\left(Z-Z^{\prime}\right) /\left(A_{B}-A_{B}^{\prime}\right)$. This allows for example for a combined strong decay of a strangelet emitting a neutron and a pion. It might well be that single hadron decay is not possible while multiple hadron decay is due to shell effects. 
Note that this procedure is different from the one used in 25] where mesonic decays and multiple hadron emissions have not been implemented. Nevertheless, the authors allowed also for weak neutron decay which we will discuss later in a wider class (section $\nabla$ ) separately.

A strangelet is called metastable in the following if its energy lies under the corresponding (free) hadronic matter of the same baryon number, charge, and strangeness, and if it can not emit a single hadron or multiple hadrons by strong processes as described above. A metastable strangelet can then only decay via weak decays like the nonleptonic (hadronic) decays. Strangelets which are stable against strong decay but unstable against nonleptonic weak decay will be denoted in the following as short-lived (see section [V]). Estimates of this weak decay rate range from $\tau_{n l} \approx 10^{-6}-10^{-5} \mathrm{~s}\left[26\right.$ ] and $\tau_{n l} \approx 3 \times 10^{-7} \mathrm{~s}$ [27] to anything between $\tau_{n l}=10^{-5}-10^{-10} \mathrm{~s}$ [28]. These estimates were calculated for the process $u+s \leftrightarrow d+s$ in infinite matter and depend sensitively on the difference of the $d-$ and $s$-quark chemical potentials. For the lightest strangelet, the $\mathrm{H}$-dibaryon with quark content (uuddss), one gets a lifetime of about $\tau_{n l}=10^{-8}-10^{-6} \mathrm{~s}$ depending on its mass [29].

Weak nonleptonic or hadronic decay is implemented by the hadronic decay processes mentioned above allowing for strangeness violation of $\Delta S= \pm 1$. A strangelet stable against all these weak decays can then only decay via leptonic decay of the form $s \leftrightarrow u \mathrm{e}^{-} \bar{\nu}_{e}$ and $d \leftrightarrow u \mathrm{e}^{-} \bar{\nu}_{e}$ or via radiative decays through $u s \leftrightarrow u d \gamma$. These decay modes are suppressed by the three-body space of the leptonic decay or by the electromagnetic coupling constant of the radiative decay compared to the nonleptonic decays. Both decay modes are supposed to yield similar lifetimes [23] which are then higher than the nonleptonic ones. A strangelet stable with respect to weak hadronic decay but unstable with respect to weak leptonic or radiative decay is called long-lived (see section $\nabla$ ). It lives on the time scale of the weak leptonic decay $\tau=\tau_{l}$, which has been estimated to be $\tau_{l}=10^{-4}-10^{-5} \mathrm{~s}$ in infinite matter [2, 30,26]. If a strangelet demonstrates to be stable against these decays also, then it can only decay by higher order weak decay $(\Delta S= \pm 2)$, which results in lifetimes of days (see e.g. the estimate of the $\Delta S= \pm 2$ decay of the $\mathrm{H}$-dibaryon [29]). In this case the strangelet would be super long-lived. 


\section{MEMOS}

The (multi-) strange baryonic objects are treated within the framework of an extended relativistic mean-field theory. Although the application of the mean-field approximation seems doubtful for small systems, it has been shown that the model furnishes quite remarkable results for nuclei with baryon numbers as small as $A_{B}=4$ [6]. In addition to the well-known $\sigma$ - and $\omega$-meson, strange scalar and vector mesons are introduced, the $\sigma^{*}-$ and the $\phi$-meson $\left(m_{\sigma^{*}}=975 \mathrm{MeV}, m_{\phi}=1020 \mathrm{MeV}\right)$. The latter couple to strangeness only, thus incorporating the seemingly strong attractive hyperon-hyperon interaction [7].

The vector coupling constants are chosen according to SU(6)-symmetry, the scalar coupling constants are fixed to hypernuclear data. Within this model, MEMOs consisting of combinations of $\left\{p, n, \Lambda, \Xi^{0}, \Xi^{-}\right\}$baryons demonstrate to be metastable due to Pauli-blocking effects. They possess binding energies per baryon of $E_{B} / A_{B} \approx-22 \mathrm{MeV}$, strangeness per baryon of up to $f_{s} \approx 2$, unusual charge per baryon of $f_{z} \approx-0.5$ to zero while carrying positive baryon number and baryon densities up to $2.5-3$ times that of ordinary nuclei. Metastable clusters of purely hyperonic matter $\left\{\Lambda, \Xi^{0}, \Xi^{-}\right\}$have been also predicted [7].

In the following we extend the calculation of [7] to small mass numbers relevant for heavy ion physics. We calculate even combinations of $\left\{\mathrm{n}, \mathrm{p}, \Lambda, \Xi^{0}, \Xi^{-}\right\}$baryons up to a filled s- and p-shell, i.e. 8 baryons of each using model 2 of Ref. [7]. Out of these 3125 combinations we have found 298 configurations, which are bound and metastable. The two smallest systems for $A_{B}=4$ are ${ }^{4} \mathrm{He}$ and the corresponding $\Xi$-system, i.e. two $\Xi^{-}$and two $\Xi^{0}$. The next heavier ones $\left(A_{B}=6\right)$ are the combinations ${ }_{\Lambda \Lambda}^{6} \mathrm{He},\left\{2 \mathrm{n}, 2 \Lambda, 2 \Xi^{-}\right\},\left\{2 \mathrm{p}, 2 \Lambda, 2 \Xi^{0}\right\}$, and $\left\{2 \Lambda, 2 \Xi^{0}, 2 \Xi^{-}\right\}$. Pure $\Lambda$ or neutron matter is not bound in this model. Nevertheless, we have found some very loosely bound $\left\{\Xi^{0}, \Xi^{-}\right\}$-systems. The properties of these small MEMOs are summarized in Table [1]. Note that the double $\Lambda$ hypernucleus ${ }_{\Lambda}^{6} \mathrm{He}$ has already been seen [31]. Other light candidates are discussed extensively in [7], like $\Xi^{0} \Xi^{0} \mathrm{He}$ and $\Lambda \Lambda \Xi^{7} \mathrm{He}$.

The binding energy per baryon for these small systems is not more than $-16 \mathrm{MeV}$ for 
baryon numbers less than $A_{B} \approx 16$. For higher baryon numbers we have found an approximately linear decrease of the binding energy. This fact is already known from ordinary nuclei. The less bound combinations found are mainly combinations of $\Xi^{-}$and $\Xi^{0}$, i.e. pure $\Xi$-matter with only $E_{B} / A_{B} \approx-2 \mathrm{MeV}$.

MEMOs decay by weak mesonic or nonmesonic decay in analogy to hypernuclear weak decay. The nonmesonic decays of the type $\Lambda N \rightarrow N N, \Xi N, \Lambda \Lambda \rightarrow \Lambda N, \Sigma N, \ldots$ will play an important rôle [7]. Due to the high mass differences involved in these decays (about $180-200 \mathrm{MeV}$ ) the process is not hindered by Pauli-blocking effects. The first process has been seen in the weak decay of hypernuclei, which decay on the time scale of the lifetime of

the $\Lambda$, i.e. $\tau_{w} \approx \tau_{\Lambda} \approx 10^{-10}$ s. Also the mesonic decay $\Lambda \rightarrow N \pi$ yields similar lifetimes for very light hypernuclei (see e.g. [32]). We expect that MEMOs will live then on the same time scale, $\tau_{\text {MEMO }} \approx \tau_{w}$, irrespective of their strangeness content, if they are not deeper bound to create a minimum in the total energy at finite strangeness as it is the case for strangelets.

\section{SHORT-LIVED STRANGE MATTER}

In the following we will discuss the properties of light strangelets and compare them to those of MEMOs.

Fig. 1 shows the energy per baryon of all possible quark bags with a baryon number up to $A_{B}=40$ as a function of the strangeness per baryon $f_{s}=|S| / A_{B}$ for a bag constant of $B^{1 / 4}=170 \mathrm{MeV}$. We only show bags with equal numbers of up and down quarks. The energetically most favourable combinations always have the same number of up and down quarks since they occupy the same single-particle levels. The Coulomb correction is in the order of some $\mathrm{MeV}$ and thus not important in our case.

The solid line connects the masses of the nucleon, $\Lambda, \Xi$ and $\Omega$. As a first cut for potential candidates, quark bags lying above this line can (and probably will) completely decay via strong processes, those lying beneath the line will lead in principle to metastable strangelets. The important point to note here is that any strangelet initially formed with a mass under 
this line might decay to another strangelet changing baryon number, strangeness and charge, but can not decay to a pure hadronic state anymore, simply because of energy conservation! It is remarkable that there exists a quite sharp lower limit of the binding energies: already for bags with $A_{B} \approx 40$ infinite quark matter is quite a good approximation.

It is clear that this simple version of the MIT-Bag model is not able to reproduce the hadron spectrum. The masses of the nucleon, $\Lambda$ and $\Xi$ are overestimated, the mass of the $\Omega$ is underestimated. To provide a better description of the hadron masses, one-gluon exchange corrections and a zero-point energy term of the form $Z_{0} / R$ must be taken into account [33. Strangelets with s-states only up to a mass number of $A_{B}=6$ have been studied extensively in [34 taking into account colourmagnetic and colourelectric corrections. No strangelet has been found to be metastable with the exception of the $\mathrm{H}$-dibaryon with $A_{B}=2, S=-2$ and $Z=0$. This finding has been confirmed in [22]. Up to now, these corrections can only be applied to quarks sitting in s-states. We are not aware of any attempt including these terms for higher shell states also.

For a bag constant of $B^{1 / 4}=145 \mathrm{MeV}$ (not shown) almost all bags are lighter than the corresponding hyperonic matter. After subsequent strong and weak processes the final resulting strangelets thus should have long enough lifetimes to be detectable. Nevertheless, for the situation depicted in Fig. 1 $\left(B^{1 / 4}=170 \mathrm{MeV}\right)$ strangelets with strangeness fraction less than $f_{s} \approx 0.6$ are less bound than corresponding hyperonic matter (solid line) and hence, they can completely decay strongly into hyperons and nucleons and are not detectable in heavy ion searches. For intermediate strangeness fractions only bags with rather large baryon number then can be stable with respect to strong decay. This means that there exists a minimum critical strangeness fraction for which strangelets exist which will depend of course on the bag parameter and baryon number of the strangelet.

The dependence on the baryon number and the bag parameter is illustrated in the following. We have calculated the mass of all possible bags with $A_{B} \leq 16$, which are most interesting for heavy ion experiments, but with arbitrary numbers of up, down and strange quarks and various bag parameters. As already stated, we define metastable strangelets as 
those who are stable against strong decay, while unstable strangelets are not.

Figs. 20 show the strangeness and the charge fraction versus the baryon number for bag parameters of $B^{1 / 4}=150,160,170 \mathrm{MeV}$, respectively. Dots stand for metastable, open circles for unstable bags and crosses for small MEMOs. For higher bag parameters, one sees less candidates of metastable strangelets and they are shifted to higher strangeness, higher masses and, more important, to negative charges!

For $B^{1 / 4}=150 \mathrm{MeV}$ shown in Fig. 2, metastable strangelets exist for a wide range of charge $(|Z| / A \leq 1)$ and strangeness fraction. Only for quite low strangeness fraction $f_{s}<0.4$, comparable to the ones of light double $\Lambda$ hypernuclei, strangelets are unstable with respect to strong decay. This situation changes when looking at the case of $B^{1 / 4}=160$ $\mathrm{MeV}$ depicted in Fig. 3. Nearly no strangelet appears to be metastable with $f_{s}<1$ or $Z / A>0.6$ in the mass range considered here as they are subject to fissioning into nucleons and hyperons. Especially for light systems, the maximum charge is $Z=+2$. This trend is getting even more pronounced for $B^{1 / 4}=170 \mathrm{MeV}$ as can be seen in Fig. 4 . Only a few of the very light strangelets for $A \leq 6$ remain to be metastable. They are highly negatively charged $Z / A_{B}<-0.5$ and have a very high strangeness fraction of $f_{s}>2.5$. For higher baryon numbers $\left(6<A_{B}<16\right)$ no metastable strangelet exists for $f_{s}<1$ and $Z / A>0.5$. Most of the metastable strangelets are found to be highly negatively charged which is contrary to the conclusion drawn in [25]. Here the authors start their consideration in the minimum for a given baryon number due to flavor equilibrium. Nevertheless, a hot strangelet formed in heavy ion collision does not start decaying with flavor changing decays but with strong decays. Our results demonstrate that the cascade of strong decays of a strangelet do not stop in an absolute minimum but in a region allowing for highly charged strangelets.

The hadronic counterpart, MEMOs, are also shown as crosses in figs. 2 for comparison. There are candidates which are highly positive and negative charged $(-0.6<Z / A<+0.7)$. MEMOs show up where strangelets are unstable and vice versa. There is also a region in the $f_{s}-Z / A$ plane where both MEMOs and strangelets appear. Here, the energetically least 
favourable object will decay into the other. A strangelet created in a quark-gluon plasma can then possibly decay into a MEMO via strong interactions. On the other side, MEMOs can coalesce from the hot and hyperon-rich zone of a relativistic heavy ion collision first and form a strangelet which is then detected. The density distributions within both objects resemble each other closely. Transition matrix elements are to first approximation proportional to the overlap of both wavefunctions. Therefore, the energetically least favourable state may be a doorway state, decaying rapidly into the favourable state. In [35] the decay for a double hypernucleus to a $\mathrm{H}$-dibaryon was estimated to happen at $\tau=10^{-18}-10^{-20} \mathrm{~s}$ if the masses of the $\mathrm{H}$-dibaryon is close to $2 m_{\Lambda}$. We expect similar time scales for the transition of one form of strange matter to the other if the masses are similar. Of course, this is speculation and can not be clarified by our present knowledge of strange matter.

\section{LONG-LIVED STRANGE MATTER}

The properties of strange matter as discussed in the previous section apply for systems living on the time scale of weak interactions, i.e. $\tau_{\mathrm{MEMO}} \approx 10^{-10} \mathrm{~s}$ and $\tau_{n l}=10^{-5}-10^{-10} \mathrm{~s}$. Present experiments are looking for long-lived strangelets $\tau_{\exp }>10^{-6}-10^{-8} \mathrm{~s}$. They are not able to see MEMOs and possibly most of the strangelet candidates, if the nonleptonic decay is too fast. Nevertheless, it is known that the leptonic decay gives longer lifetimes due to the reduced phase space of the three body decay [2, 20,26. As $\tau_{l}=10^{-4}-10^{-5} \mathrm{~s}$ in infinite matter, the present experiments are sensitive to strangelets which are stable against weak nonleptonic decay. In the following we study all possible weak hadronic decay for the metastable strangelets, as weak pion, kaon, proton, neutron, $\Lambda, \Sigma^{+,-}, \Xi^{0,-}, \Omega^{-}$decays.

For example a weak neutron decay of a strangelet (which turns out to be one of the most dominant decay modes)

$$
Q\left(A_{B}, S, Z\right) \rightarrow Q\left(A_{B}-1, S-1, Z\right)+n
$$

happens if the energy balance of the corresponding reaction reads 


$$
E\left(A_{B}, S, Z\right)>E^{\prime}\left(A_{B}-1, S-1, Z\right)+m_{n}
$$

where $E$ stands for the total energy of a strangelet. Note that weak neutron decay drives a strangelet to lower strangeness fraction only if the initial strangeness content of the strangelet is $f_{s}<1$, but to higher strangeness fraction for $f_{s}>1$ as the obtained shift in the strangeness content is easily found to read $\Delta f_{s}=\left(f_{s}-1\right) /(A-1)$ and thus gets positive in this case!

Multiple hadron emission and fission to another strangelet is also checked in analogy to the strong decays described in section $\llbracket$ but now with $\Delta S= \pm 1$. In addition we add the case with $B^{1 / 4}=145 \mathrm{MeV}, m_{s}=280 \mathrm{MeV}$ which are the original MIT values [33].

We find that the strangelets mainly decay via weak pion or baryon emission. In a very few cases weak multiple hadron emission is possible. We have also checked the case $B^{1 / 4}=180 \mathrm{MeV}$ but none of the strangelets are stable with respect to weak hadronic decay. Nevertheless, for the other cases we found some candidates. The remaining long-

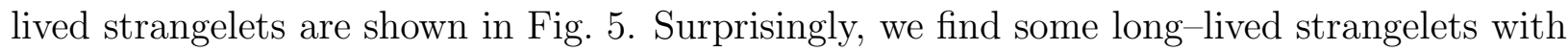
quite low mass numbers for all cases considered here. They are lying on a chain which starts from the triple magic strangelet $(6 u 6 d 6 s)$ where all quarks fill up the 1s-state - due to its symmetry character it is also called the quark alpha [36]. The 'valley of stability' starting at the quark alpha continues then towards negative charges by adding one unit of negative charge when going to a higher mass number. The reason for the stability line is a pronounced shell effect. These strangelets mainly have a closed s-shell for the $u$-quarks and a closed s-, $\mathrm{p} 3 / 2-$ or $\mathrm{p} 1 / 2-$ shell for the $s$-quarks. Then the $d$-quarks added result in the chain seen in Fig. 5. This rule is less stringent for the case $B^{1 / 4}=150 \mathrm{MeV}$ as many strangelets demonstrate to be long-lived. For the case $B^{1 / 4}=145 \mathrm{MeV}$ also strangelets with a closed $\mathrm{p} 3 / 2-$ shell for the $u$-quarks appear resulting in the positively charged candidates at $A_{B}=13,14$. Due to the higher strange quark mass of $m_{s}=280 \mathrm{MeV}$ strangelets with high strangeness fraction are less stable and only the $1 \mathrm{~s}$-shell for the $s$-quarks are filled here. Also for $B^{1 / 4}=150 \mathrm{MeV}$ there are several cases with a filled $\mathrm{p} 3 / 2$-shell for the $u$-quarks, like $A=10, Z=+8(18 u 6 d 6 s)$. The candidates shown for $A_{B} \leq 6$ have to be taken with some 
care as colourmagnetic and colourelectric terms are not included in the present investigation. If included, they appear to be not stable at all [34,22 and can decay to ordinary hadrons via strong interactions. The lightest long-lived candidates for $A_{B} \geq 6$ are summarized in Table —. We add also the candidates which can only decay by weak multiple hadron emission as the decay is suppressed by phase space. Actually, we find only one additional strangelet of this type.

Most promising candidates are for $A_{B}=10, Z=-4$, for $A_{B}=12, Z=-6$ and $A_{B}=16$, $Z=-10$, which appear in all the cases studied here. Note that the first candidate is triple magic $(6 u 6 d 18 s$ or $6 u 18 d 6 s)$.

The question arises, why mainly negatively charged strangelets appear to be stable against weak hadronic decay (and also already, to a somewhat lesser extent, against strong hadronic decay). The reason is twofold: (a) Strangelets with a rather low strangeness fraction (and correspondingly positive charges) are decaying strongly by fissioning into nucleons and hyperons as discussed in the last section. As $Z / A>0$ follows model independent from $f_{s}<1$ for isospin saturated matter this will always happen if there is a minimum energy for strangelets at high strangeness fraction. Still, in addition, any strong decay by emitting a neutron (cf. (5)) (or a proton) will enhance the relative strangeness content of the remaining strangelet by an amount $\Delta f_{s}=f_{s} /(A-1)>0$ shifting it to a higher strangeness fraction $f_{s}$ which thus can exceed 1. (b) It is easier for positively charged strangelets to decay via e.g. weak proton decay. The charge reverse reaction would be a weak neutron decay accompanied by $\pi^{-}$emission which is less favourable. In general, (the dominant) weak nucleon decay (cf. (9)) will drive strangelets with $f_{s}>1$ to a higher strangeness fraction and hence to higher negative $Z / A$-ratios.

These two reasonings should be generally valid if the masses of finite droplets of SQM follow a distribution closely to that shown in Fig. —. Although calculated within the MIT bag model, we believe that a similar distribution would in principle also show up when applying other bag models (and fixing the same overall energy scale within the appropriate parameters). In this sense our major result, i.e. the tendency of short-lived and long-lived 
metastable strangelets to exist preferably as a slightly or highly negative exotic state of matter, should be seen to be valid on more general grounds.

We want to stress again that the cascade of weak decays of a strangelet is trapped in a deep local minimum where it can only decay via weak semileptonic and radiative decay. We have checked the stability with respect to these two decays. In only two cases a strangelet demonstrates to be stable against these decays: this is the quark alpha for $B^{1 / 4}=150 \mathrm{MeV}$ and $B^{1 / 4}=160 \mathrm{MeV}$, and the strangelet $(6 u 6 d 3 s)$ with $A_{B}=5, Z=+1$ for $B^{1 / 4}=150$ $\mathrm{MeV}$. They would live on the time scale of days as only weak decays with $\Delta S= \pm 2$ are allowed. Due to its nonzero charge only the later would be visible in heavy ion experiment. Nevertheless, the colourmagnetic term is repulsive for these multiquark states. For the quark alpha, the colourmagnetic term is about $+150 \mathrm{MeV} / \mathrm{A}$ without symmetry breaking effects, i.e. half the mass splitting of the nucleon and Delta. Therefore, the colourmagnetic interaction would shift the mass above the mass of $6 \Lambda$ 's. Then, the quark alpha is simply a resonance.

It is interesting to study now the detectability of these long-lived strangelets in heavy ion collisions especially for $A_{B}>6$. All recent experiments searching for strangelets are sensitive to composites with lifetimes of $\tau>10-100 \mathrm{~ns}$ except for experiment NA52 with $\tau>10^{-6} \mathrm{~s}$.

Experiment E814 [8] was looking for positively charged strangelets with $0.1<Z / A<0.3$, $A_{B}>10$. Their limit on the production of a strangelet in a single event was $1.2 \times 10^{-4}$ for multiply charged strangelets. Experiment E886 was set up to $0<A / Z<14$ yielding a much higher sensitivity of down to $10^{-7}$ /event [11]. An open geometry is used by experiment E864 which reported new limits for $Z=+1,+2$ and a wide range of mass $A_{B}>10$ of about $10^{-5}-10^{-6}$ event just recently [12]. According to Fig. 5 these experiments could see the positively charged candidates for $B^{1 / 4}=145 \mathrm{MeV}\left(m_{s}=280 \mathrm{MeV}\right)$ at $A_{B}=13,14,16$ and the ones for $B^{1 / 4}=150 \mathrm{MeV}$ at $A_{B}=14-16$.

The high sensitivity experiment E858 [9] was looking in the range $-1<A / Z<-7$ and no strangelet was found with a sensitivity of $10^{-9}-10^{-10}$ /event. Also the follow-up 
experiment E878 [10] using the gold beam did not see any evidence for unusual composites with $|Z| \leq 3$ on the level of $10^{-7} /$ event. Nevertheless, these experiments were measuring at zero degree and were not measuring at midrapidity for $A_{B}>8$ [10. Hence, they are only sensitive to e.g. the candidate $A_{B}=7, Z=-1$ for the case $B^{1 / 4}=150 \mathrm{MeV}$ and for the other charged candidates at $A_{B}<6$ for $B^{1 / 4}=150,160 \mathrm{MeV}$.

Unfortunately, none of these experiments has set limits so far for the other candidates in the valley of stability, like $A_{B}=10$ and $Z=-4$ and for higher negative charges. While finishing this work, new results from E886 were published which give limits for negatively charged strangelets down to $10^{-8} /$ event, but unfortunately for $|Z|<4$ only [11]. Most recently, experiment NA52 presented new limits for negatively charged strangelets with a sensitivity down to $10^{-8}-10^{-10}$ /event for $M /|Z|=10-40$ [13]. However, their limit for the above mentioned strangelets with $M /|Z| \approx 2$ is much less, about $10^{-6}-10^{-7}$ /event. Moreover, results were presented for rigidities of $p / Z=40,100,200 \mathrm{GeV}$ only, while NA52 can cover the range of $p / Z=5-200 \mathrm{GeV}$. Midrapidity is reached for $M / Z \approx p / 9 Z$ [13] corresponding to $M / Z=4.4 \mathrm{GeV}$ for the lowest rigidity of $p / Z=40 \mathrm{GeV}$ measured so far. Lower rigidities are therefore probing strangelets with lower $M / Z$ and NA52 would be sensitive to the negatively charged strangelet candidates proposed here.

\section{SUMMARY}

The present investigation seems to indicate that the search for highly charged strange matter would be far more promising than hitherto recognized. Most search experiments are, however, designed to detect only particles with small charge-to-mass ratio. But long-lived positively charged strangelets seems to exist only for $A_{B}>12$ and very low bag parameters. The most interesting candidates for long-lived strangelets are lying in a valley of stability which starts at the quark alpha $(6 u 6 d 6 s)$ and continues by adding one unit of negative charge, i.e. $(A, Z)=(8,-2),(9,-3),(10,-4),(11,-5) \ldots$ The present experimental setups are hardly sensitive to these candidates. Plans for extending experiment E864 to look for 
highly charged strangelets for $A_{B} \geq 10$ are therefore most interesting [37].

On the other hand, experiments looking for short-lived strange matter will be able to see a much wider variety of combinations of charge and mass. Recently, experiment E896 started looking into this rich domain of short-lived composites for the $\mathrm{H}$-dibaryon [17] but other composites with low mass-to-charge ratios might be also accessible. Short-lived strange matter, either in the form of metastable strangelets or MEMOs, demonstrate to be also highly negatively charged which opens the possibility for measuring their formation with an extremely low background from antinuclei. These metastable composites can be detected by a cascade of weak decays and by their unusual charge and mass. Measuring single and double $\Lambda$ hypernuclei will set limits on the production possibility of MEMOs. This limit can possibly also be applied for strangelets as MEMOs can decay to them via strong decay and serve as a doorway state. The other way around, if MEMOs are found they will set stringent limits on the existence of strangelets for the same charge, strangeness and mass and will give new impetus for our understanding of the strong interactions between baryons in general.

\section{ACKNOWLEDGMENTS}

This paper is dedicated to our former collaborator, Carl B. Dover. We thank H. Crawford, R. Klingenberg, J. Nagle, K. Pretzl, F. Rotondo, J. Sandweiss for useful discussions and remarks. J.S.B. thanks the Niels Bohr Institute for the warm hospitality and the Alexander von Humboldt-Stiftung for its support with a Feodor-Lynen fellowship. This work was supported in part by the Graduiertenkolleg 'Theoretische und Experimentelle Schwerionenphysik' of the Deutsche Forschungsgemeinschaft (DFG), the Gesellschaft für Schwe-

rionenforschung Darmstadt (GSI) and the Bundesministerium für Bildung und Forschung $(\mathrm{BMBF})$. 


\section{REFERENCES}

[1] A.R. Bodmer, Phys. Rev. D 4, 1601 (1971)

[2] S.A. Chin and A.K. Kerman, Phys. Rev. Lett. 43, 1292 (1979)

[3] E. Witten, Phys. Rev. D 30, 272 (1984);

[4] C. Greiner, H. Stöcker, P. Koch, Phys. Rev. Lett. 58, 1825 (1987); C. Greiner, D.H. Rischke, H. Stöcker and P. Koch, Phys. Rev. D 38, 2797 (1988)

[5] C. Greiner and H. Stöcker, Phys. Rev. D 44, 3517 (1991)

[6] J. Schaffner, C. Greiner, H. Stöcker, Phys. Rev. C 46, 322 (1992)

[7] J. Schaffner, C.B. Dover, A. Gal, C. Greiner, H. Stöcker, Phys. Rev. Lett. 71, 1328 (1993); J. Schaffner, C.B. Dover, A. Gal, D.J. Millener, C. Greiner, H. Stöcker, Ann. Phys. (N.Y.) 235, 35 (1994)

[8] E814 Collaboration, J. Barrette et al., Phys. Lett. B252, 550 (1990); F. Rotondo et al., Nucl. Phys. B (Proc. Suppl.) 24B, 265 (1991)

[9] E858 Collaboration, M. Aoki et al., Phys. Rev. Lett. 69, 2345 (1992); H. Crawford et al., Nucl. Phys. B (Proc. Suppl.) 24B, 251 (1991)

[10] E878 Collaboration, D. Beavis et al., Phys. Rev. Lett. 75, 3078 (1995)

[11] E886 Collaboration, A. Rusek et al., Nucl. Phys. A585, 59c (1995); Phys. Rev. C 54, R15 (1996)

[12] E864 Collaboration, J. Sandweiss, Nucl. Phys. B (Proc. Suppl.) 24B, 234 (1991); F. Rotondo, talk given at the quark matter conference ' 96 in Heidelberg, to be published in Nucl. Phys. A

[13] NA52 (NEWMASS) Collaboration, K. Borer et al., Phys. Rev. Lett. 72, 1415 (1994); F. Dittus et al., Nucl. Phys. A590, 347c (1995); R. Klingenberg, private communication 
and talk given at the quark matter conference '96 in Heidelberg, to be published in Nucl. Phys. A

[14] R.L. Jaffe, Phys. Rev. Lett. 38, 195 (1977); 617 (E)

[15] C.B. Dover, Nucl. Phys. A450, 95c (1986); C.B. Dover, P. Koch, M. May, Phys. Rev. C 40, 115 (1989) and references therein

[16] J. Belz et al., Phys. Rev. Lett. 76, 3277 (1996)

[17] H. Crawford, private communication and talk given at the quark matter conference '96 in Heidelberg, to be published in Nucl. Phys. A

[18] R. Mattiello, C. Hartnack, A.v. Keitz, J. Schaffner, H. Sorge, H. Stöcker, C. Greiner, Nucl. Phys. B (Proc. Suppl.) 24B, 221 (1991); M. Sano, M. Wakai, Czech. J. Phys. 42, 1239 (1992); A.J. Baltz, C.B. Dover, S.H. Kahana, Y. Pang, T.J. Schlagel, E. Schnedermann, Phys. Lett. B325, 7 (1994); L. Gerland, C. Spieles, M. Bleicher, P. Papazoglou, J. Brachmann, A. Dumitru, H. Stocker, W. Greiner, J. Schaffner, C. Greiner, nucl-th/9512032

[19] H.W. Barz, B.L. Friman, J. Knoll, H. Schulz, Nucl. Phys. B (Proc. Suppl.) 24B, 211 (1991)

[20] C. Spieles, L. Gerland, H. Stöcker, C. Greiner, C. Kuhn, J.P. coffin, Phys. Rev. Lett. 76, 1776 (1996); C. Spieles, M. Bleicher, L. Gerland, H. Stöcker, C. Greiner: Proceedings of International Conference on Nuclear Physics at the Turn of Millennium: Structure of Vacuum and Elementary Matter, George, South Africa, 10-16 March 1996, nuclth/9606027

[21] ALICE Technical Proposal, CERN/LHCC 95-71

[22] E. Farhi and R.L. Jaffe, Phys. Rev. D 30, 2379 (1984)

[23] M.S. Berger and R.L. Jaffe, Phys. Rev. C 35, 213 (1987); 44, 566(E) (1991) 
[24] J. Madsen, Phys. Rev. Lett. 70, 391 (1993); Phys. Rev. D 47, 5156 (1993); 50, 3328 (1994)

[25] E.P. Gilson and R.L. Jaffe, Phys. Rev. Lett. 71, 332 (1993)

[26] P. Koch, Nucl. Phys. B (Proc. Suppl.) 24B, 255 (1991)

[27] H. Heiselberg, Phys. Scr. 46, 485 (1992)

[28] J. Madsen, Phys. Rev. D 47, 325 (1993)

[29] J.F. Donoghue, E. Golowich, B.R. Holstein, Phys. Rev. D 34, 3434 (1986)

[30] H. Heiselberg, J. Madsen, K. Riisager, Phys. Scr. 34, 556 (1986)

[31] D.J. Prowse, Phys. Rev. Lett. 17, 782 (1966)

[32] J.J. Szymanski et al., Phys. Rev. C 43, 849 (1991) and references therein

[33] T. DeGrand, R.L. Jaffe, K. Johnson, J. Kiskis, Phys. Rev. D 12, 2060 (1975)

[34] A.Th.M. Aerts, P.J.G. Mulders and J.J. de Swart, Phys. Rev. D 17, 260 (1978)

[35] B.O. Kerbikov, Sov. J. Nucl. Phys. 39, 516 (1984)

[36] F.C. Michel, Phys. Rev. Lett. 60, 677 (1988)

[37] J. Sandweiss, private communication 


\section{TABLES}

TABLE I. Some candidates for long-lived strangelets with $A \geq 6$ which are stable against weak nonleptonic decay. Case I: $B^{1 / 4}=145 \mathrm{MeV}, m_{s}=280 \mathrm{MeV}$; Case II: $B^{1 / 4}=150 \mathrm{MeV}$, $m_{s}=150 \mathrm{MeV}$; Case III: $B^{1 / 4}=160 \mathrm{MeV}, m_{s}=150 \mathrm{MeV}$; Case IV: $B^{1 / 4}=170 \mathrm{MeV}, m_{s}=150$ $\mathrm{MeV} .{ }^{*}$ This candidate can decay via a collective nonleptonic weak decay.

\begin{tabular}{cccccccccccccc}
\hline \hline $\mathrm{A}$ & 6 & 7 & 8 & 8 & 9 & 9 & 10 & 10 & 11 & 12 & 12 & 13 & 13 \\
\hline $\mathrm{Z}$ & 0 & -1 & $-8,-3^{*}$ & -2 & $-4,-5,-6$ & -3 & -4 & $-3,+8$ & -5 & -6 & -5 & -7 & $+2,3,4,5$ \\
\hline case & I-III & II & II & I-III & II & I-III & I-IV & II & I-III & I-IV & II & II-IV & I \\
\hline \hline
\end{tabular}

TABLE II. Candidates of small MEMOs for filled s-shell states. Here we only consider $\left(\operatorname{pn} \Lambda \Xi^{0} \Xi^{-}\right)$baryons. The double hypernucleus ${ }_{\Lambda \Lambda}^{6} \mathrm{He}$ which was already seen [31] belongs also to this class. Candidates involving $\Sigma$ baryons are $2\left(\mathrm{n} \Sigma^{-} \Xi^{-}\right)$and $2\left(\mathrm{p} \Sigma^{+} \Xi^{0}\right)$.

\begin{tabular}{|c|c|c|c|c|c|c|c|c|c|}
\hline $2(3$ & $\left.0^{0} \Xi^{-}\right)$ & $2\left(\Lambda \Xi^{0} \Xi^{-}\right)$ & $2\left(\mathrm{n} \Lambda \Xi^{-}\right)$ & $2\left(\mathrm{p} \Lambda \Xi^{0}\right)$ & $2\left(\mathrm{n} \Lambda \Xi^{0} \Xi^{-}\right)$ & $2\left(\mathrm{p} \Lambda \Xi^{0} \Xi^{-}\right)$ & $2\left(\operatorname{pn} \Lambda \Xi^{-}\right)$ & $2\left(\operatorname{pn} \Lambda \Xi^{0}\right)$ & $2\left(\operatorname{pn} \Lambda \Xi^{0} \Xi^{-}\right)$ \\
\hline A & 4 & 6 & 6 & 6 & 8 & 8 & 8 & 8 & 10 \\
\hline $\mathrm{Z}$ & -2 & -2 & -2 & +2 & -2 & 0 & 0 & +2 & 0 \\
\hline S & 8 & 10 & 6 & 6 & 10 & 10 & 6 & 6 & 10 \\
\hline
\end{tabular}




\section{FIGURES}

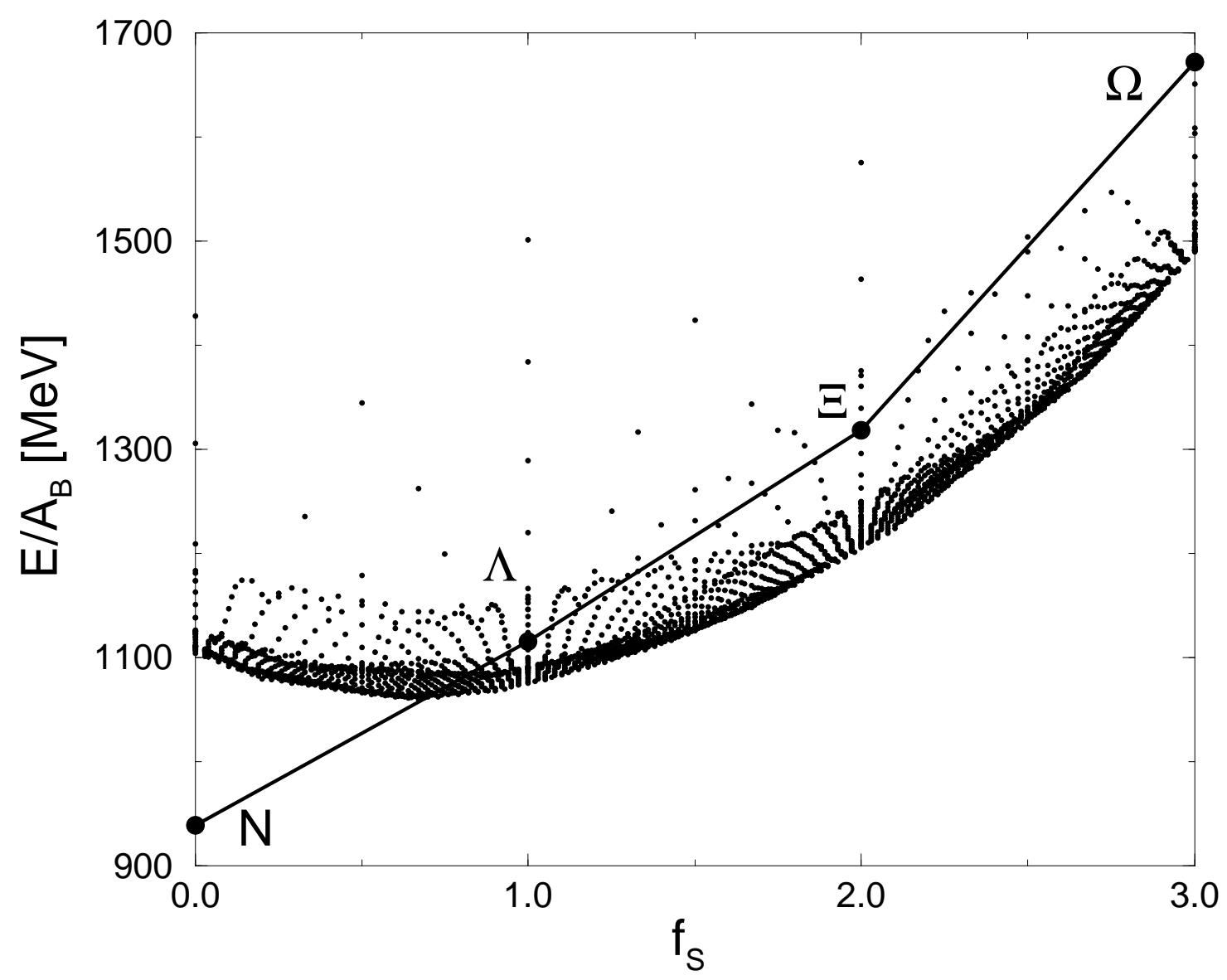

FIG. 1. The energy per baryon $E / A_{B}$ of all possible strangelets with $A_{B} \leq 40$ and $N_{u}=N_{d}$ for a bag constant of $B^{1 / 4}=170 \mathrm{MeV}$ versus the strangeness fraction $f_{s}$. The solid line connects the masses of nucleon, $\Lambda, \Xi$ and $\Omega$ and stands for free baryon matter. 


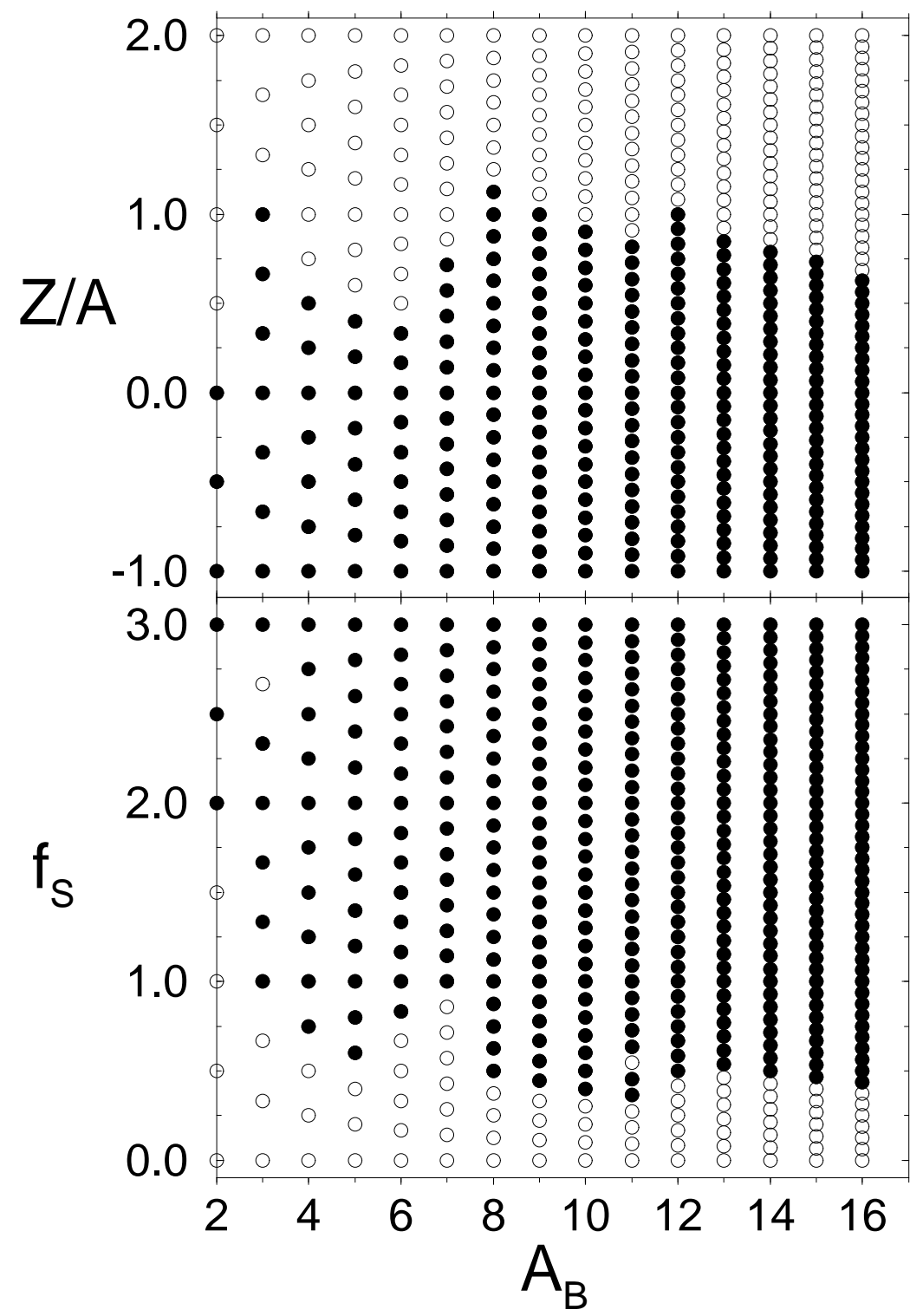

FIG. 2. The strangeness per baryon $f_{s}$ (lower part) and the charge fraction $Z / A$ (upper part) as a function of the baryon number $A_{B}$ for short-lived strangelets (dots), unstable strangelets (open circles) for a bag constant of $B^{1 / 4}=150 \mathrm{MeV}$. The hadronic counterpart, MEMOs, are shown by crosses. 


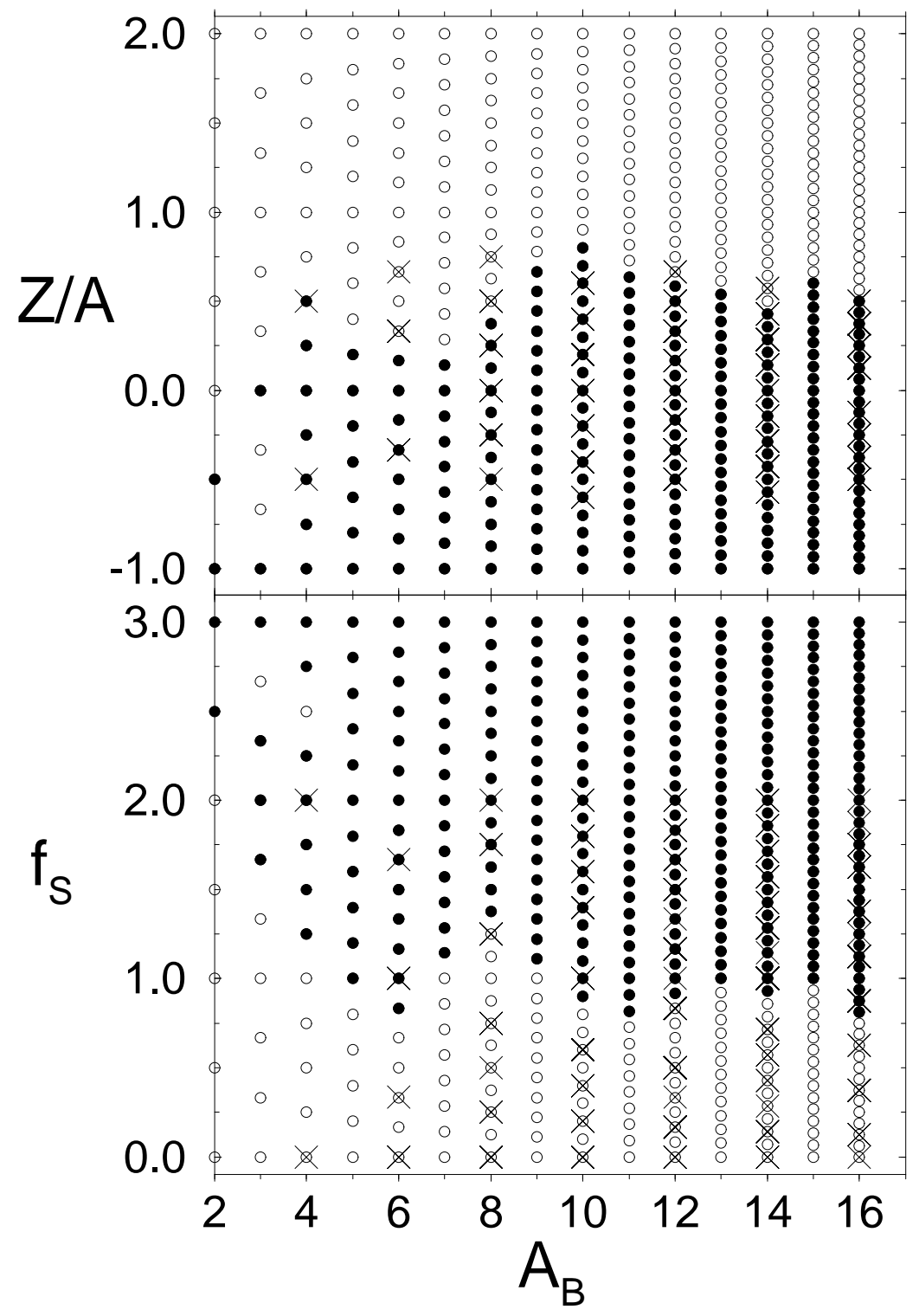

FIG. 3. The same as Fig. 2 for a bag constant of $B^{1 / 4}=160 \mathrm{MeV}$. 


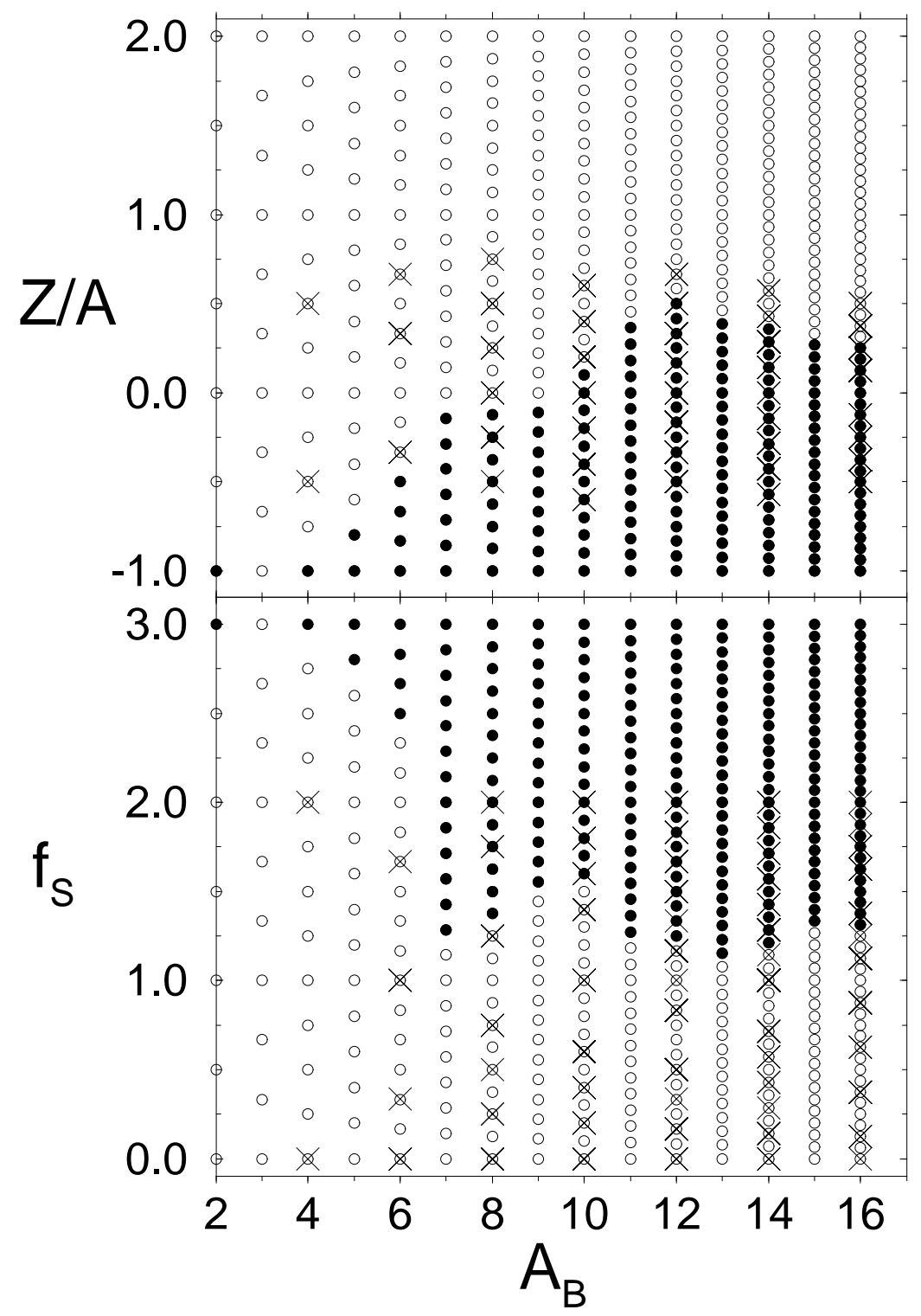

FIG. 4. The same as Fig. 2 for a bag constant of $B^{1 / 4}=170 \mathrm{MeV}$. 


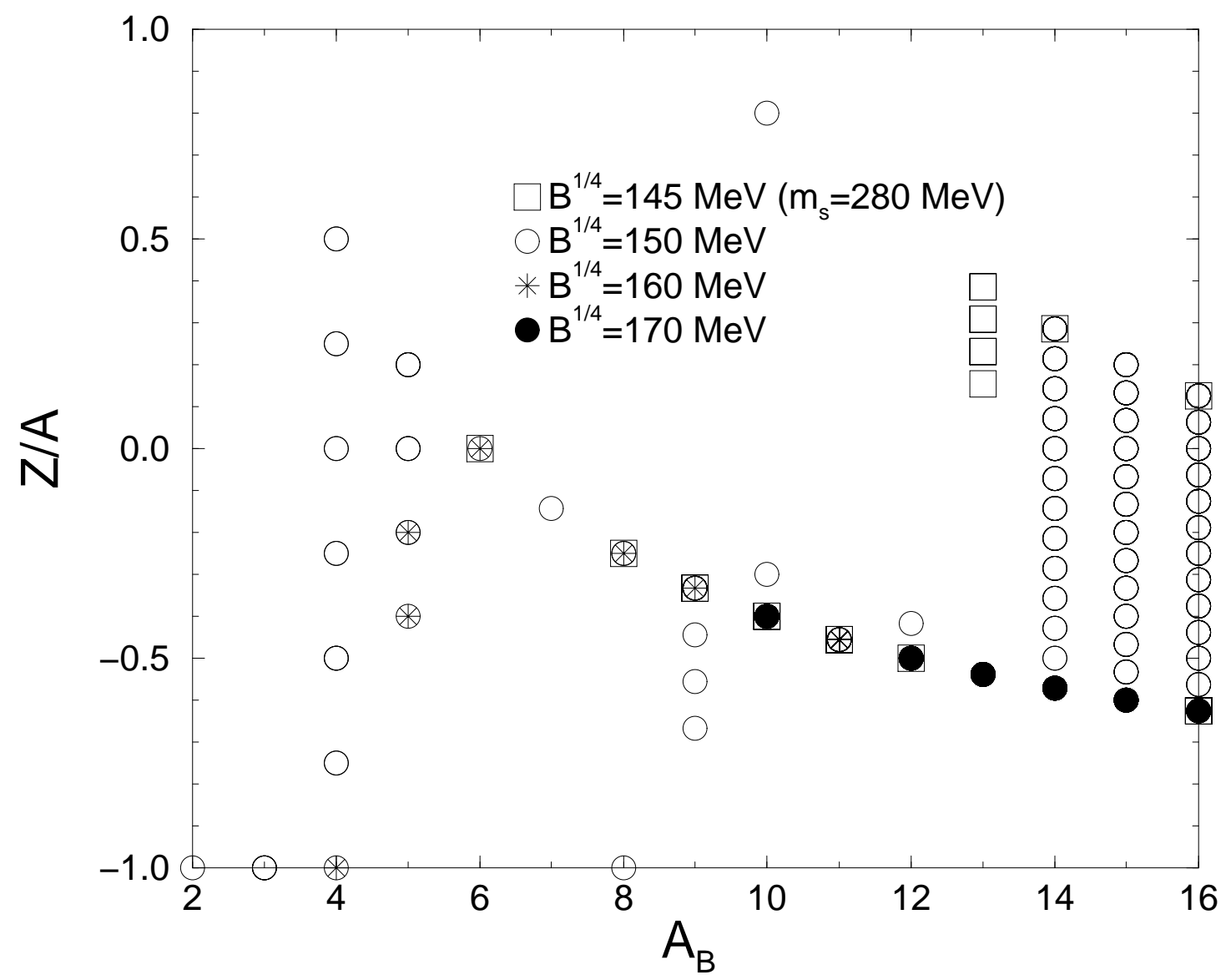

FIG. 5. The charge fraction $Z / A$ for long-lived strangelets, which are stable against nonleptonic weak decay, for different choices of the bag parameter. The case for the original MIT parameters $\left(B^{1 / 4}=145 \mathrm{MeV}, m_{s}=280 \mathrm{MeV}\right)$ is also shown. 